\title{
Implantation Temperature Effects on the Nanoscale Optical Pattern Fabrication in a-SiC:H Films by $\mathrm{Ga}^{+}$Focused Ion Beams
}

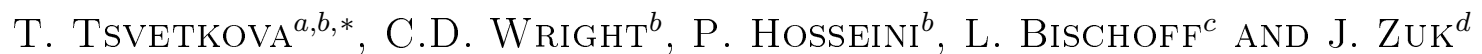 \\ ${ }^{a}$ Institute of Solid State Physics, Bulgarian Academy of Sciences, 72 Tzarigradsko Chaussee, 1784 Sofia, Bulgaria \\ ${ }^{b}$ University of Exeter, College of Engineering, Mathematics and Physical Sciences, Harrison Building \\ North Park Rd, Exeter EX4 4QF, UK \\ ${ }^{c}$ Institute of Ion Beam Physics and Materials Research, Helmholtz-Zentrum Dresden-Rossendorf \\ P.O.B. 5101 19, 01314 Dresden, Germany \\ ${ }^{d}$ Institute of Physics, Maria Curie-Skłodowska University, pl. M. Curie-Skłodowskiej 1, 20-031 Lublin, Poland
}

\begin{abstract}
This work is related to a novel approach of providing some new generation ultrastable ( $>50$ years), ultrahigh density (>1 Tbit/sq.in.) data storage for archival applications. We used ion-implantation to write nanoscale data into hydrogenated amorphous silicon carbide (a-SiC:H) films. Wide bandgap a-SiC:H samples, $\mathrm{Ga}^{+}$focused ion beam implanted, have been prepared. A range of samples has been focused ion beam patterned under different implantation conditions, with emphasis on different substrate temperatures (typically from $0{ }^{\circ} \mathrm{C}$ temperature to around room temperature). Some of the room temperature implanted samples were further annealed at $+250{ }^{\circ} \mathrm{C}$ in vacuum. The focused ion beam patterned samples were then analysed using near-field techniques, like atomic force microscopy, to define optimum implantation conditions and the resulting consequences for archival data storage applications. The atomic force microscopy analysis of $\mathrm{Ga}^{+}$focused ion beam implanted a-Si ${ }_{1-x} \mathrm{C}_{x}: \mathrm{H}$ samples at room temperature and at $0{ }^{\circ} \mathrm{C}$ revealed an increase of both the depth and the width of the individual lines within the focused ion beam written patterns at the lower temperature, as a result of an increased ion beam induced sputtering yield, in good agreement with the previous results for the case of $\mathrm{Ga}^{+}$broad beam implantation in a-Si ${ }_{1-x} \mathrm{C}_{x}: \mathrm{H}$ and again suggesting that the best conditions for optical data storage for archival storage applications would be using $\mathrm{Ga}^{+}$ion implantation in a-SiC:H films with an optimal dose at room temperatures. Similarly, the atomic force microscopy results confirm that no advantage is expected to result from post-implantation annealing treatments.
\end{abstract}

DOI: 10.12693 /APhysPolA.123.952

PACS: 85.40.Hp, 81.16.Rf, 68.55.Ln

\section{Introduction}

Hydrogenated amorphous silicon-carbon (a-SiC:H) alloy films have been studied for a long time as materials with a wide range of useful optical properties (e.g. high transparency in the visible region due to their wide optical bandgap), as well as mechanical durability and chemical inertness [1]. These properties, together with the thermal stability and simple fabrication, make $\mathrm{a}-\mathrm{SiC}: \mathrm{H}$ very promising for various optoelectronic device applications in adverse environments $[2,3]$. Radio-frequency $(\mathrm{RF})$ reactive magnetron sputtering is a convenient method for preparation of $\mathrm{a}-\mathrm{SiC}: \mathrm{H}$ films where low density-of-states material is not required, as is often the case [4].

The use of ion beam techniques is a very attractive method to control material properties via introducing foreign elements into a host material in order to modify its chemical composition and hence the electronic characteristics, such as the band-gap width and related optical

*corresponding author; e-mail: tania_tsvetkova@yahoo.co.uk and electrical properties [5, 6]. This method is of particular interest in relation to the emerging technology of computer-operated focused ion beam (FIB) systems, and the possibilities they provide for fabrication of various sophisticated planar structures [7-9]. Promising results have been obtained when implementing this technique to develop a new method for high-density optical data storage and sub- $\mu \mathrm{m}$ lithographic masks production using a-SiC:H films [10-13].

The use of gallium as the ion implanted species is particularly attractive since it is available in standard FIB machines, and in addition has been shown to be capable of generating large optical contrasts $[14,15]$. The fact that $\mathrm{Ga}$ has a very low melting point $\left(T_{\mathrm{m}}=29.80^{\circ} \mathrm{C}\right)$ and an unusual feature of volume contraction on melting are the factors which favour $\mathrm{Ga}$ incorporation upon ion-implantation as dispersed clusters, or small nanoparticles. It was previously noted that Ga precipitation into nanoparticles can vary dramatically (in terms of particle size) with $\mathrm{Ga}$ concentration and small changes in the surface implant temperature [16]. The precise role of implantation temperature effects, i.e. the target temperature during $\mathrm{Ga}^{+}$ion irradiation and appropriate post-implantation annealing treatments at different temperatures, were studied with respect to optical con- 
trast achieved [17] and obtained data storage densities/ feature size (present work) so that to optimize the cost-effectiveness of the FIB bit-writing method.

\section{Experimental}

Thin a-Si $\mathrm{Si}_{1-x} \mathrm{C}_{x}$ :H films $(x=0.18)$ were deposited onto the Corning glass substrates by $\mathrm{RF}(13.56 \mathrm{MHz})$ reactive magnetron sputtering. A composite target, composed of monocrystalline (100) silicon wafer with chips of pure graphite placed on it, was sputtered in the $\mathrm{Ar}-20 \% \mathrm{H}_{2}$ gas mixture. The typical deposition conditions are RF power $150 \mathrm{~W}$ (power density $1.91 \mathrm{~W} / \mathrm{cm}^{2}$ ), total gas pressure $1 \mathrm{P}$, substrate temperature $275^{\circ} \mathrm{C}$, and graphite-to-silicon target ratio 0.025 . The film thickness was determined by the Talystep profilometer and optical measurements to be $\approx 200 \mathrm{~nm}$. The Rutherford backscattering spectrometry (RBS) was used to determine the carbon content $(x)$ of the films.

FIB implanted wide bandgap a-SiC:H samples were prepared at Helmholtz-Zentrum Dresden-Rossendorf (HZDR), Germany. The FIB system at HZDR was a CANION 31Mplus column, Orsay Physics, working with $\mathrm{Ga}^{+}$ions, and was used to create sub-micrometre optical patterns in a-SiC:H films at different target temperatures. Focused $\mathrm{Ga}^{+}$irradiation in these samples was performed with the FIB system while also simultaneously employing a charge neutralizer (electron-beam shower) to implant a series of patterns with different ion doses in the range $1 \times 10^{15}-1.25 \times 10^{17}$ ions $\mathrm{cm}^{-2}$. The choice of the $\mathrm{Ga}^{+}$ion dose range has been prompted by earlier results, where the optimised range of ion doses for $\mathrm{Ga}^{+}$and other elements has been established to be the same so that to yield a good optical contrast. The chosen type of the combined implanted pattern consisted of combination of 4 individual patterns: a full square, an open square and 2 sets of parallel lines, situated around a central cross-feature (designed for an eye-guide in the microscope of the AFM so that to find the area of the nano-scale pattern at the start of measurements) (Fig. 1).

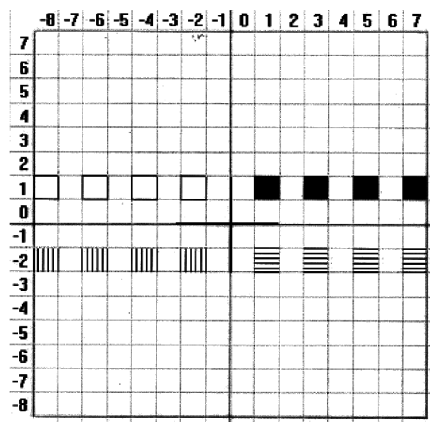

Fig. 1. Characteristic FIB pattern.

Atomic force microscope (Dimension 3000 Digital Instruments) (AFM) [11] was used to analyze the topography of the fabricated patterned samples. A lab-built scanning near-field optical microscope (SNOM) $[11,18]$ was utilized to study the morphology and existing non-homogeneities in the as-deposited a-SiC:H films.

\section{Results and discussion}

The as-deposited and some broad-beam $\mathrm{Ga}^{+}$ion implanted samples were analyzed applying high-resolution optical imaging using SNOM. Imaging was performed using a range of different wavelengths $(\lambda=500 \div 800 \mathrm{~nm})$, including the wavelength of the $\mathrm{He}-\mathrm{Ne}$ laser used for the further SNOM analysis of the $\mathrm{Ga}^{+}$FIB patterned a-SiC:H/glass samples $(\lambda=633 \mathrm{~nm})$. The obtained results showed that both the as-deposited and the ion-implanted samples exhibit sub- $\mu \mathrm{m}$ scale non-uniformity with the regions of lower and higher optical absorptions, presumably due to variations in samples stochiometry in the sub- $\mu \mathrm{m}$ scale, as shown below (Fig. 2) for the case of the a-Si $\mathrm{Si}_{1-x} \mathrm{C}_{x}: \mathrm{H}\left(x_{2}=0.35\right)$ sample implanted with $\mathrm{Ga}^{+}$with a dose $D=5 \times 10^{16} \mathrm{~cm}^{-2}$.
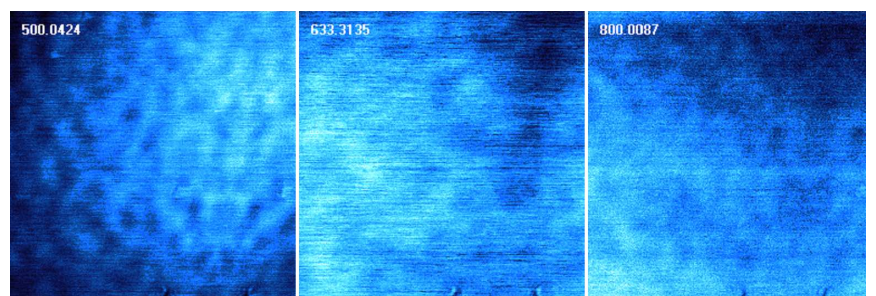

Fig. 2. High-resolution SNOM optical imaging $(25 \times$ $\left.25 \mu \mathrm{m}^{2}\right)$ of an a-Si $\mathrm{S}_{1-x} \mathrm{C}_{x}: \mathrm{H}\left(x_{2}=0.35\right)$ sample implanted with $\mathrm{Ga}^{+}$with a dose $D=5 \times 10^{16} \mathrm{~cm}^{-2}$.

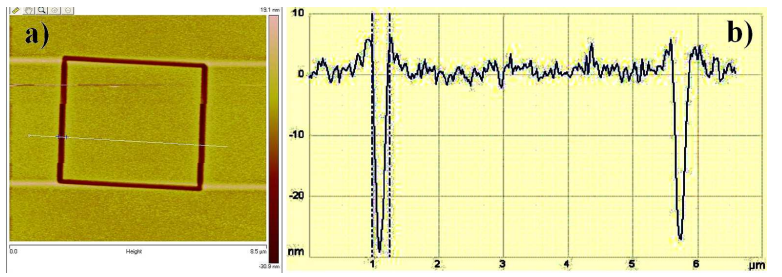

Fig. 3. AFM topographic image (a) and cross-section (b) of a FIB patterned open square in an a-Si ${ }_{1-x} \mathrm{C}_{x}: \mathrm{H}$ $\left(x_{2}=0.35\right)$ sample with a $\mathrm{Ga}^{+}$ion dose $D_{4}=$ $1.25 \times 10^{17} \mathrm{~cm}^{-2}$.

The actual patterns consisted of combination of the above described individual patterns, each of which was implanted at 4 different doses: $1 \times 10^{15} \mathrm{~cm}^{-2}$, $5 \times 10^{15} \mathrm{~cm}^{-2}, 2.5 \times 10^{16} \mathrm{~cm}^{-2}$, and $1.25 \times 10^{17} \mathrm{~cm}^{-2}$. For this purpose a specially designed programme was used with the FIB equipment at HZDR. The length of the individual lines in each pattern as well as the side of the full and open squares thereof, were fixed to be $5 \mu \mathrm{m}$, while all line-widths were $200 \mathrm{~nm}$.

Some low temperature $\left(0^{\circ} \mathrm{C}\right)$ implanted samples were prepared, while a greater number of RT implanted samples were designed with view of further post-implantation 
annealing treatments for the investigation of their effect on the recorded optical pattern characteristics, to be studied there using near-field techniques (AFM).

AFM topographic analysis of the FIB implanted samples has been focused mainly on the open squares patterns (example in Fig. 3). AFM results for RT and $0^{\circ} \mathrm{C}$ implanted samples revealed an increased ion beam induced sputtering yield for the $0{ }^{\circ} \mathrm{C}$ implanted samples as compared to the RT implanted ones, as previously noted during the $\mathrm{Ga}^{+}$broad beam implantation studies. In the $\mathrm{Ga}^{+}$FIB case, this resulted in an increase of both the depth and the width of the individual lines within the patterns (Table).

Depth $(h)$ and width $(w)$ of the lines in the $\mathrm{Ga}^{+}$FIB written open square patterns in a-Si ${ }_{1-x} \mathrm{C}_{x}: \mathrm{H}$.

\begin{tabular}{c|c|c|c|c|c|c|c|c|c}
\hline \hline \multirow{2}{*}{$\mathrm{a}-\mathrm{Si}_{1-x} \mathrm{C}_{x}: \mathrm{H}$} & \multicolumn{2}{|c|}{$D_{1}=1 \times 10^{15} \mathrm{~cm}^{-2}$} & \multicolumn{2}{c|}{$D_{2}=5 \times 10^{15} \mathrm{~cm}^{-2}$} & \multicolumn{2}{c|}{$D_{3}=2.5 \times 10^{16} \mathrm{~cm}^{-2}$} & \multicolumn{2}{c}{$D_{4}=1.25 \times 10^{17} \mathrm{~cm}^{-2}$} \\
\cline { 2 - 10 } & $h_{1}[\mathrm{~nm}]$ & $w_{1}[\mu \mathrm{m}]$ & $h_{2}[\mathrm{~nm}]$ & $w_{2}[\mu \mathrm{m}]$ & $h_{3}[\mathrm{~nm}]$ & $w_{3}[\mu \mathrm{m}]$ & $h_{4}[\mathrm{~nm}]$ & $w_{4}[\mu \mathrm{m}]$ \\
\hline \multirow{2}{*}{$x_{1}=0.18$} & $\mathrm{RT}$ & 3 & 0.25 & 4 & 0.30 & 4 & 0.70 & 4.5 & 0.85 \\
& $0{ }^{\circ} \mathrm{C}$ & 3 & 0.59 & 9 & 0.69 & 11 & 0.79 & 12 & 0.99 \\
\hline \multirow{2}{*}{$x_{2}=0.35$} & $\mathrm{RT}$ & 2.8 & 0.15 & 3 & 0.16 & 8 & 0.17 & 10 & 0.19 \\
& $0{ }^{\circ} \mathrm{C}$ & 9 & 0.22 & 15 & 0.25 & 18 & 0.27 & 22 & 0.50
\end{tabular}

Some of the RT $\mathrm{Ga}^{+}$FIB implanted a-Si ${ }_{1-x} \mathrm{C}_{x}: \mathrm{H}$ samples have been thermally annealed in vacuum at $T=$ $250{ }^{\circ} \mathrm{C}$. The AFM topographic analysis of the RT FIB implanted and the thermally annealed samples has been focused again on the open squares patterns (Fig. $4-$ the examples for the highest doses in the a- $\mathrm{Si}_{0.65} \mathrm{C}_{0.35}: \mathrm{H}$ samples). The AFM results for the RT implanted and the thermally annealed samples revealed slight changes in the depth of the individual lines within the FIB written patterns. The noticeable decrease of the depth of the individual lines after the thermal annealing, as seen in Fig. 4, and hence obtained slight thickness increase in the irradiated areas of the films, which should contribute to an increased optical absorption thereof, and hence result in an increased optical contrast as compared to the non-irradiated film areas, is expected to be overridden by the reduced absorption in the irradiated areas, as a result of the thermal annealing of the ion beam induced irradiation defects, as observed in the broad-beam implanted case [17].

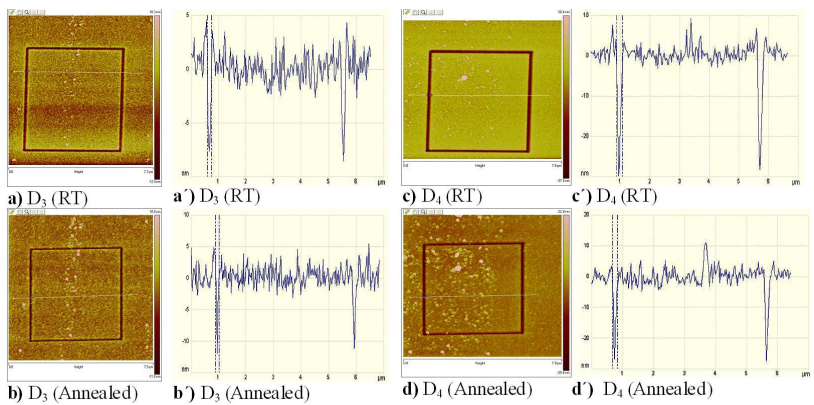

Fig. 4. AFM topographic images of $\mathrm{Ga}^{+}$FIB patterned open square patterns in a- $\mathrm{Si}_{1-x} \mathrm{C}_{x}: \mathrm{H}(x=0.35)$ samples with doses $D_{3}=2.5 \times 10^{16} \mathrm{~cm}^{-2}$, before (a) and after annealing (b), and $D_{4}=1.25 \times 10^{17} \mathrm{~cm}^{-2}$, before (c) and after annealing (d), together with their corresponding cross-sections $\left(a^{\prime}, b^{\prime}, c^{\prime}\right.$ and $\left.d^{\prime}\right)$.

\section{Conclusions}

The AFM analysis of the $\mathrm{Ga}^{+}$FIB implanted a-Si $i_{1-x} \mathrm{C}_{x}: \mathrm{H}$ samples at $\mathrm{RT}$ and at $0{ }^{\circ} \mathrm{C}$ revealed an increase of both the depth and the width of the individual lines within the FIB written patterns at the lower temperature, as a result of an increased ion beam induced sputtering yield, in good agreement with the previous results for the case of $\mathrm{Ga}^{+}$broad beam implantation in a-Si $i_{1-x} \mathrm{C}_{x}: \mathrm{H}$ and again suggesting that the best conditions for optical data storage for archival storage applications would be using $\mathrm{Ga}^{+}$ion implantation in a-SiC:H films with an optimal dose of $D=5 \times 10^{16} \mathrm{~cm}^{-2}$ at room temperatures. Similarly, the AFM results confirm that no advantage is expected to result from post-implantation annealing treatments.

\section{Acknowledgments}

This work has been supported by the European Community as an Integrating Activity "Support of Public and Industrial Research Using Ion Beam Technology (SPIRIT)" under EC contract No. 227012. The support of EC funded project BG051PO001/3.3.-05.001 for this publication is gratefully acknowledged. The Marie Curie Fellowship for T. Tsvetkova was also supported by the European Community under the contract PIEF-GA-2009-251845. The help of D. Dimova-Malinovska and O. Angelov with the samples preparation and useful discussions is also gratefully acknowledged.

\section{References}

[1] J. Bullot, M.P. Schmidt, Phys. Status Solidi B $\mathbf{1 4 3}$, 345 (1987).

[2] J.A. Powell, L. Matus, in: Amorphous and Crystalline Silicon Carbide, Eds. G.L. Harris, C.Y.W. Yang, Springer, Berlin 1989. 
[3] Amorphous and Microcrystalline Semiconductor Devices, Ed. J. Kanicki, Artech House, Boston 1991.

[4] T. Nagai, H. Yamamoto, I. Kobayashi, J. Phys. E 15, 520 (1982).

[5] J.K. Hirvonen, Ion Implantation and Ion Beam Processing of Materials, North Holland, Amsterdam 1984.

[6] J.F. Ziegler, Ion Implantation, Academic Press, New York 1988.

[7] K. Böhringer, K. Jousten, S. Kalbitzer, Nucl. Instrum. Methods Phys. Res. B 30, 289 (1988).

[8] B. Ruttensperger, G. Krötz, G. Müller, G. Derst, S. Kalbitzer, J. Non-Cryst. Solids 137-138, 635 (1991).

[9] G. Müller, Nucl. Instrum. Methods Phys. Res. B 80-81, 957 (1993).

[10] T. Tsvetkova, in: Beam Processing of Advanced Materials, Eds. J. Singh, S. Copley, J. Mazumder, ASM International, Metals Park 1996, p. 207.

[11] T. Tsvetkova, S. Takahashi, A. Zayats, P. Dawson, R. Turner, L. Bischoff, O. Angelov, D. DimovaMalinovska, Vacuum 79, 94 (2005).

[12] T. Tsvetkova, S. Takahashi, A. Zayats, P. Dawson, R. Turner, L. Bischoff, O. Angelov, D. DimovaMalinovska, Vacuum 79, 100 (2005).
[13] S. Takahashi, P. Dawson, A.V. Zayats, L. Bischoff, O. Angelov, D. Dimova-Malinovska, T. Tsvetkova, P.D. Townsend, J Phys. D, Appl. Phys. 40, 7492 (2007).

[14] L. Bischoff, J. Teichert, S. Kitova, T. Tsvetkova, Vacuum 69, 73 (2002).

[15] T. Tsvetkova, O. Angelov, M. Sendova-Vassileva, D. Dimova-Malinovska, L. Bischoff, G.J. Adriaenssens, W. Grudzinski, J. Zuk, Vacuum 70, 467 (2003).

[16] D.E. Hole, P.D. Townsend, J.D. Barton, L.C. Nistor, J. Van Landuyt, J. Non-Cryst. Sol. 180, 266 (1995).

[17] T. Tsvetkova, C.D. Wright, S. Kitova, L. Bischoff, J. Zuk, Nucl. Instrum. Methods Phys. Res. B, 2013, to be published.

[18] Nano-Optics and Near-Field Microscopy, Eds. D. Richards, A.V. Zayats, Philos. Trans. R. Soc. A 362, 699 (2004). 